That doesn't excuse any case, says Stratmann, adding that he is committed to a zero-tolerance approach to both issues. The survey comes as the international academic community grapples with issues of bullying, which have emerged in the past year or so. However, many academic institutions have not had formal bullying policies or definitions of the behaviour. Stratmann says that the MPS is creating a code of conduct for bullying in response to the results, and is rolling out mandatory training.

The survey also found that more nonscientific staff members than scientific ones felt that they had been bullied - 23\% compared with $13 \%$. And $14 \%$ of women said they had experienced sexual harassment in a period longer than the past 12 months. But unexpectedly, says Stratmann, women in leadership positions reported experiencing sexist behaviour at a higher rate than others - $26 \%$ of directors and group leaders, compared with $23 \%$ of postdocs and $25 \%$ of $\mathrm{PhD}$ students.

"The Max Planck survey shows an

\section{WORKING CULTURE}

The Max Planck Society in Germany conducted a huge social study of its staff and received more than 9,000 responses. The results will inform the development of policies on topics including bullying.

\section{Have you been bullied?}

- In past 12 months In more than a year

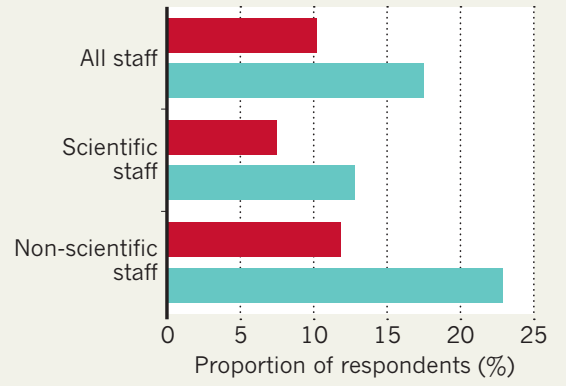

apparently lower level of bullying than other academic surveys - but what really matters is that they say that the level they observe is unacceptable, and plan to do something about it," says Loraleigh Keashly, an occupational psychologist at Wayne State University in Detroit, Michigan, who specializes in workplace bullying.

Different people - for example, those from different cultures - might not consider the same types of behaviour to be bullying. So the survey asked respondents whether they had experienced particular behaviours that are indicated in the social-science literature to be bullying, such as having opinions ignored or being unfairly blamed, publicly humiliated or shouted at. Around 60\% reported having experienced one or more such behaviours.

The MPS's international make-up was another focus of the survey: $36 \%$ of research directors there are from outside Germany, as are $75 \%$ of postdocs. But $45 \%$ of the non-Germans working at MPS institutes felt excluded. "We have to be really worried about this," says Stratmann, who speculates that one reason for this could be language barriers.

\title{
US opioid crisis is driving a spike in infectious diseases
}

\section{Researchers around the country are scrambling to understand and identify the outbreaks.}

\section{BY SARA REARDON}

$\mathrm{O}$ pioid addiction kills tens of thousands of people in the United States every year, and the trend shows no signs of slowing. Now, public-health officials are worried about a surge in bacterial and viral infections linked to opioid misuse that threatens to compound the crisis.

The surge includes an unprecedented rise in bacterial infections - including those caused by Staphylococcus aureus, a bacterium that's frequently resistant to antibiotics and a spike in new cases of HIV and hepatitis associated with opioid use that risks undoing decades of progress against these diseases.

Research groups around the country are working to understand, identify and treat the outbreaks. But the lack of solid data on the number of new cases, and where they'll crop up next, as well as the stigma associated with drug use that can prevent people with infections from seeking early treatment, is hindering efforts.

"This is like HIV all over again," says Judith Feinberg, an infectious-disease physician at West Virginia University in Morgantown, comparing the current crisis to the HIV epidemic that dominated US public-health efforts in the 1980 and 1990s. "People are stigmatized; they don't feel they deserve to live. They hear people say it's a lifestyle choice."

Over the past 20 years, the use of opioids, including prescription pain medications, heroin and synthetic drugs such as fentanyl, has skyrocketed in the United States. In 2017, there were roughly 15 opioid-overdose-related deaths per 100,000 people in the country, compared with 3 per 100,000 in 1999, according to estimates from the US Centers for Disease Control and Prevention.

One type of opioid-related infection that

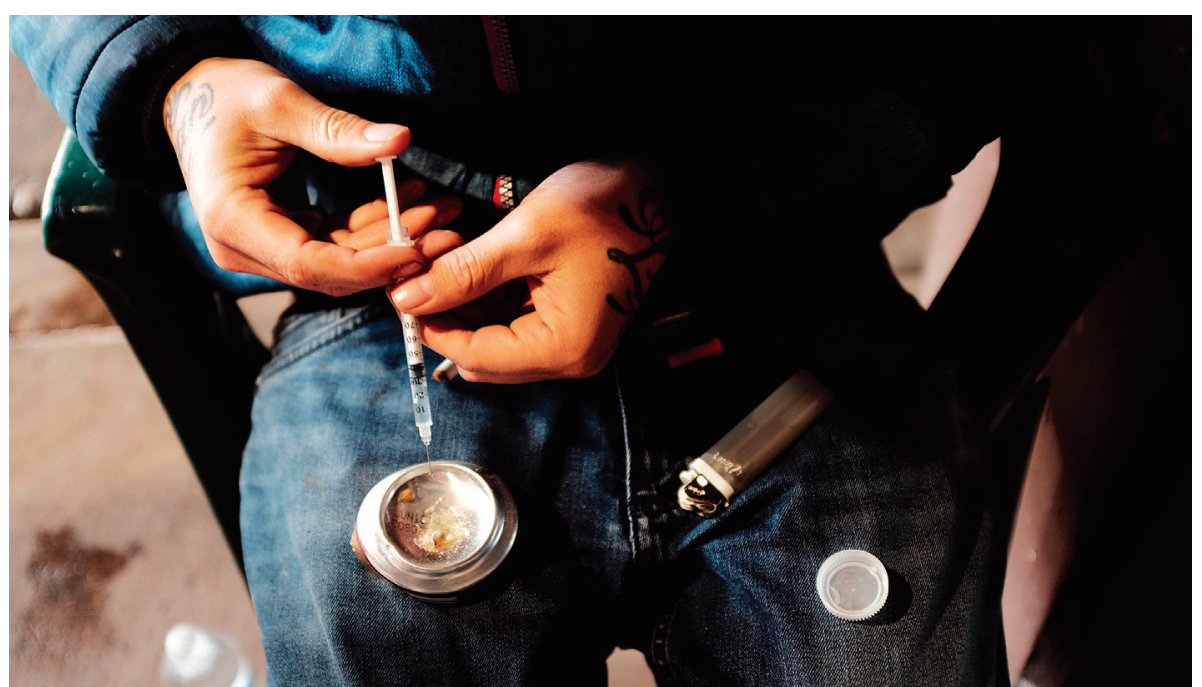

Misuse of opioids such as heroin has led to a surge in diseases including HIV, risking years of progress . 
- researchers are grappling with involves diseased heart valves. Bacteria such as $S$. aureus can enter the bloodstream as a result of practices such as needle sharing or not cleaning the skin before injecting a drug. If the infection reaches the heart, it can damage the valves. Severe cases can require a heart transplant.

In an ongoing study, microbiologist Cecilia Thompson at the University of North Carolina in Chapel Hill is sequencing DNA from heart valves collected from people who have had surgery to replace diseased valves with artificial ones. Thompson has found that valves taken from people who had injected drugs are more likely to be infected with $S$. aureus than are those of people who don't use drugs.

Thompson presented her results at the American Society for Microbiology meeting in San Francisco, California, on 21 June. But these are just the latest observations of what seems to be a worrying trend. In a study published in January ${ }^{1}$, researchers found a 13 -fold increase in heart infections among drug users in North Carolina between 2007 and 2017. Until 2013, surgeons in the state performed fewer than 10 operations annually to treat drug-related heart infections, compared with more than 100 in 2017.

Opioids themselves - rather than the method used to inject them - could also be making people more susceptible to infection.
Another study ${ }^{2}$, also published in January, looked at more than 25,000 people treated at veterans' health facilities between 2000 and 2012. It found that people who took medium or high doses of prescribed opioids for pain management were significantly more susceptible to pneumonia. It's unclear why, but research in monkeys suggests that some prescription opioids, such as morphine, suppress the immune system ${ }^{3}$.

\section{IT'S ABOUT RELATIONSHIPS}

Researchers also struggle with anticipating the location of outbreaks associated with drug use, because the patterns can differ from those of non-drug-related ones.

But a computer model developed by Georgiy Bobashev, a data scientist at RTI International, a non-profit research institute in Research Triangle Park, North Carolina, and his colleagues simulates drug users and their social networks to predict where outbreaks of HIV infections might occur. The program considers factors including whom users know, the type of heroin available to them - which could affect the presence of pathogens - and their experience with the drug.

The social component of predicting outbreak patterns is crucial, Bobashev says. People who used drugs during the height of the HIV epidemic in the 1990s learnt safe injection practices, he says, but newer users are more likely to use riskier methods, such as sharing needles.

In an unpublished analysis, Bobashev's group's model predicted that HIV outbreaks related to opioids would be concentrated in small geographic pockets, rather than spread over a wider area, as researchers would expect with non-drug-related outbreaks.

Data from real life bolster this result. Previous opioid-related HIV outbreaks, including one in 2014 in Scott County, Indiana, followed this pattern. And in March, the West Virginia health department announced an outbreak in Cabell County due to a spike in new cases of HIV acquired through drug use.

The key to stopping the rise in opioidassociated infections and halting their rise is to treat opioid use as a disease without stigmatizing drug users, says Carlos Del Rio, a global-health researcher at Emory University in Atlanta, Georgia.

A working group at the US National Academy of Medicine, which Del Rio is leading, has started to develop a strategy for integrating care for infections and opioid use. "The opioid epidemic is going to be to [young medical students] what HIV was to me," Del Rio says. "You'd better get used to it." -

1. Schranz, A. J., Fleischauer, A., Chu, V. H., Wu, L.-T. \& Rosen, D. L. Ann. Intern. Med. 170, 31-40 (2019).

2. Edelman, E. J. et al. JAMA Intern. Med. 179, 297-304 (2019).

3. Kumar, R. et al. J. Virol. 78, 11425-11428 (2004).

\section{Discrimination drives LGBT+ scientists to think about quitting}

\section{Physical scientists from sexual and gender minorities are excluded at work, finds UK survey.}

\section{BY ELIZABETH GIBNEY}

$\mathrm{N}$ early one-third of physical scientists from sexual and gender minorities in the United Kingdom have considered leaving their jobs because of their workplace climate, a survey suggests.

Some $18 \%$ of these LGBT+ scientists said they had experienced harassment, bullying or exclusionary behaviour in the workplace. That figure rises to $32 \%$ for transgender people and those who don't identify as either male or female (non-binary). Transgender and non-binary scientists were overall more likely than other the groups to describe challenges in their work environment, and women generally reported more negative experiences than men.

The findings come from a survey published on 26 June by British scientific societies that spoke to more than 600 people working in

\section{LGBT+ EXPERIENCES}

In a UK survey of LGBT+ physical scientists, non-binary and transgender people were most likely to feel uncomfortable at work and to experience exclusionary behaviour.

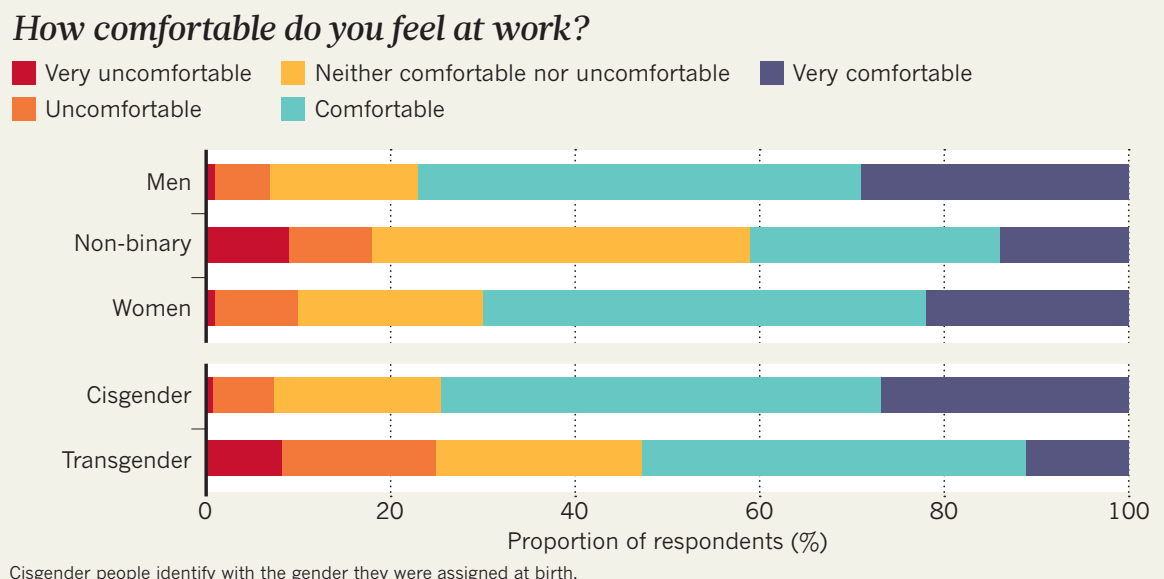

